\title{
On double Natural transform and its applications
}

\author{
Adem Kiliçman $^{a, *}$, Maryam Omran ${ }^{b}$ \\ ${ }^{a}$ Department of Mathematics and Institute for Mathematical Research, University Putra Malaysia 43400 Serdang, Selangor, Malaysia. \\ ${ }^{b}$ Institute for Mathematical Research, University Putra Malaysia 43400 Serdang, Selangor, Malaysia.
}

Communicated by A. Atangana

\begin{abstract}
In this work, we generalize the concept of one dimensional Natural transform to two dimensional Natural transform namely, double Natural transform and some of its properties are given. We also set a relation between double Natural transform and double Laplace, double Sumudu transforms. Further the convolution theorem with a proof is investigated with some details. Double Natural transform is applied to get the solutions of some general linear telegraphs, wave and partial integro-differential equations. (C) 2017 All rights reserved.
\end{abstract}

Keywords: Double Natural transform, single Natural transform, partial differential and integro-differential equations, convolution theorem.

2010 MSC: 44A30, 44A35, 35Axx, 45K05.

\section{Introduction}

Partial differential equations and their applications arise frequently in many branches of physics, engineering, and other sciences. There are many works that provided using integral transform method to solve some types of partial differential equations, for example in $[12,17]$ integral transform is used to solve boundary value problems and integral equations.

Laplace transform is one of the most used in the mathematical and engineering community. Definition of the Laplace transform, notations, and Laplace transforms of some elementary functions can be found in [28].

Furthermore, one dimensional Laplace transform was extended to two dimensional and called as double Laplace transform. The first introduction of double Laplace transform was in [17]. Some operation calculus of double Laplace transform can be found in [10]. Double Laplace transform was used to solve heat, wave, and Laplace's equations with convolution terms (see [13]), telegraph and partial integrodifferential equations (see [16]).

Sumudu transform was first introduced by [30] and some of its applications were given by [2, 14, 20, 22]. For more information and features regarding Sumudu transform we refer to [3-6].

\footnotetext{
*Corresponding author

Email addresses: akilic@upm.edu.my (Adem Kiliçman), maryamomran83@yahoo.com (Maryam Omran)
} 
Analogous to Laplace transform, double Sumudu transform was established by [29] and used to get the solution of non-homogeneous wave equation with constant coefficient (see [23]).

Additionally, [19, 24] double Laplace and Sumudu transforms were used to solve wave and Poisson equations, further the relation between them and their applications to differential equations were determined and studied by $[15,21]$.

Natural transform is closely related to Laplace and Sumudu transforms. The Natural transform was first established by [18] that was known as $\mathrm{N}$-transform and its properties were investigated by [1,9]. In $[7,26]$, the Natural transform is applied to solve Maxwell's equations. For more information we refer to [8]. Recently there was attempt to study and extend Natural transform to fractional way (see [25]).

The aims of this study are to generalize the definition of single Natural transform to double Natural transform and achieve its main properties, in order to solve telegraph, wave and partial integro-differential equations.

\section{Preliminaries}

Definition 2.1 ([28]). Laplace transform of $f(x)$ for $x>0$ is denoted by $\mathscr{L}\{f(x)\}$ or $F(s)$ and known as

$$
\mathscr{L}\{\mathrm{f}(\mathrm{x})\}=\mathrm{F}(\mathrm{s})=\int_{0}^{\infty} \mathrm{e}^{-s \mathrm{~s}} \mathrm{f}(\mathrm{x}) \mathrm{dx},
$$

where $s$ is a complex number.

Definition $2.2([17,27])$. Double Laplace transform of two variables function $f(x, y)$ for $x, y>0$ is denoted by $\mathscr{L}^{2}\{f(x, y)\}=F^{2}(s, p)$ and defined as

$$
\mathscr{L}^{2}\{\mathrm{f}(\mathrm{x}, \mathrm{y})\}=\mathrm{F}^{2}(\mathrm{~s}, \mathrm{p})=\int_{0}^{\infty} \int_{0}^{\infty} e^{-s x} e^{-p y} f(x, y) \mathrm{d} x \mathrm{~d} y, \text { where } x, y \in \mathbb{R}_{+} .
$$

Definition 2.3 ([31]). Sumudu transform of a function $f(x)$ is denoted by $\mathcal{S}\{f(x)\}=G(u)$ and defined as

$$
\mathcal{S}\{f(x)\}=G(u)=\frac{1}{u} \int_{0}^{\infty} e^{-\frac{x}{u}} f(x) d x, \text { where } x \in \mathbb{R}_{+},
$$

on the set of the functions

$$
\mathcal{A}=\left\{f(x): \exists M, \tau_{1}, \tau_{2}>0,|f(x)|<M e^{\frac{x}{\tau_{i}}}, \text { if } x \in(-1)^{i} \times[0, \infty)\right\} .
$$

Definition 2.4 ([29]). Double Sumudu transform of function $f(x, y)$ is denoted by $\mathcal{S}^{2}\{f(x, y)\}=G^{2}(u, v)$ and defined as

$$
\mathcal{S}^{2}\{f(x, y)\}=G^{2}(u, v)=\frac{1}{u v} \int_{0}^{\infty} \int_{0}^{\infty} e^{-\frac{x}{u}} e^{-\frac{y}{v}} f(x, y) d x d y, \quad \text { where } x, y \in \mathbb{R}_{+},
$$

where $\mathcal{S}^{2}$ indicates double Sumudu transform.

Definition 2.5 ([11, 17]). If $f(x, y)$ and $g(x, y)$ are integrable functions, then double convolution of $f(x, y)$ and $g(x, y)$ is given by

$$
(f * * g)(x, y)=\int_{0}^{y} \int_{0}^{x} f(\alpha, \beta) g(x-\alpha, y-\beta) d \alpha d \beta,
$$

and the symbol $* *$ denotes the double convolution respect to $x$ and $y$.

Definition $2.6([9,18])$. The Natural transform can be given by

$$
\mathbb{N}_{+}\{f(x)\}=R_{+}[f(x) ; s, u]=\frac{1}{u} \int_{0}^{\infty} e^{-\frac{x s}{u}} f(x) d x, \text { where } x \in \mathbb{R}_{+},
$$

on the set of the functions

$$
\mathcal{A}=\left\{f(x): \exists M, \tau_{1}, \tau_{2}>0,|f(x)|<M e^{\frac{x}{\tau_{i}}}, \text { if } x \in(-1)^{i} \times[0, \infty)\right\},
$$

where $s, u$ are variables of the transform. 


\section{Definition of double Natural transform and double Natural transform of some special functions}

In this part, we present double Natural transform definition and its inverse. Furthermore, we apply double Natural transform on some special functions.

Definition 3.1. Let $f(x, y)$ be a function and $x, y \in \mathbb{R}_{+}$, then double Natural transform is stated as

$$
\mathbb{N}_{+}^{2}\{f(x, y)\}:=: R_{+}^{2}[(s, p) ;(u, v)]=\int_{0}^{\infty} \int_{0}^{\infty} e^{-(s x+p y)} f(u x, v y) d x d y
$$

We can rewrite (3.1) in other form as

$$
\mathbb{N}_{+}^{2}\{f(x, y)\}=\frac{1}{u v} \int_{0}^{\infty} \int_{0}^{\infty} e^{-\left(\frac{s x}{u}+\frac{p y}{v}\right)} f(x, y) d x d y
$$

Remark 3.2. From the above definition, the equation (3.2) will turn to double Laplace and Sumudu transforms as follows.

1. when $u=v=1$, then we obtain double Laplace transform as the formula (2.1) in Definition 2.2,

2. when $s=p=1$, then we obtain double Sumudu transform as the formula (2.2) in Definition 2.4.

Definition 3.3. Double inverse Natural transform can be written as the formula

$$
\mathbb{N}_{+}^{-2}\left[\mathbb{N}_{+}^{2} f(x, y)\right]=f(x, y)=\frac{1}{2 \pi i} \int_{a-i \infty}^{a+i \infty} e^{\frac{s x}{u}} d s \frac{1}{2 \pi i} \int_{b-i \infty}^{b+i \infty} e^{\frac{p y}{v}} \mathbb{N}_{+}^{2}\{f(x, y)\} d p
$$

Now, we apply double Natural transform on some special functions as follows.

1. Let $f(x, t)=1$ for $x>0$ and $y>0$, then

$$
\mathbb{N}_{+}^{2}\{f(x, y)\}=\frac{1}{u v} \int_{0}^{\infty} \int_{0}^{\infty} e^{-\left(\frac{s x}{u}+\frac{p y}{v}\right)} d x d y=\frac{1}{s p} .
$$

2. Let $f(x, t)=x^{m} y^{n}$, where $m, n$ are positive integers, then

$$
\begin{aligned}
\mathbb{N}_{+}^{2}\{f(x, t)\} & =\frac{1}{u v} \int_{0}^{\infty} \int_{0}^{\infty} e^{-\left(\frac{s x}{u}+\frac{p y}{v}\right)} x^{m} y^{n} d x d y \\
& =\frac{1}{u} \int_{0}^{\infty} e^{-\frac{s x}{u}} x^{m} d x \frac{1}{v} \int_{0}^{\infty} e^{-\frac{p y}{v}} y^{n} d y \\
& =\frac{1}{u}\left(\frac{m ! u^{m+1}}{s^{m+1}}\right) \frac{1}{v}\left(\frac{n ! v^{n+1}}{p^{n+1}}\right)=(m !)(n !) \frac{u^{m} v^{n}}{s^{m+1} p^{n+1}} .
\end{aligned}
$$

In the particular case, when $m=n$, then

$$
\mathbb{N}_{+}^{2}\left\{(x y)^{n}\right\}=(n !)^{2} \frac{u^{n} v^{n}}{s^{n+1} p^{n+1}}
$$

3. If $c, d>-1$ are real numbers, then

$$
\mathbb{N}_{+}^{2}\left\{x^{c} y^{d}\right\}=\frac{u^{c}}{s^{c+1}} \Gamma(c+1) \cdot \frac{v^{d}}{p^{d+1}} \Gamma(d+1)
$$

It follows from Definition 3.2

$$
\mathbb{N}_{+}^{2}\left\{x^{c} y^{d}\right\}=\frac{1}{u v} \int_{0}^{\infty} \int_{0}^{\infty} e^{-\left(\frac{s x}{u}+\frac{p y}{v}\right)} x^{c} y^{d} d x d y=\frac{1}{u v}\left[\int_{0}^{\infty} x^{c} e^{-\frac{s x}{u}} d x \int_{0}^{\infty} y^{d} e^{-\frac{p y}{v}} d y\right]
$$


By putting $\alpha=\frac{s x}{u}, \beta=\frac{p y}{v}$ in the right side of the formula (3.4), then we have

$$
\mathbb{N}_{+}^{2}\left\{x^{c} y^{d}\right\}=\frac{u^{c}}{s^{c+1}} \int_{0}^{\infty} \alpha^{c} e^{-\alpha} d \alpha \frac{v^{d}}{p^{d+1}} \int_{0}^{\infty} \beta^{d} e^{-\beta} d \beta=\frac{u^{c}}{s^{c+1}} \Gamma(c+1) \cdot \frac{v^{d}}{p^{d+1}} \Gamma(d+1),
$$

where $\Gamma(\mathrm{c})$ is the Euler gamma function defined by the uniformly convergent integral

$$
\Gamma(\mathrm{c})=\int_{0}^{\infty} \alpha^{\mathrm{c}-1} e^{-\alpha} \mathrm{d} \alpha, \quad c>0 .
$$

The formula (3.3) can be used to obtain the famous integral of the product of Riemann zeta functions $\zeta(\mathrm{t})$ defined by

$$
\zeta(t)=\sum_{n=1}^{\infty} \frac{1}{n^{t}} .
$$

It turns out that

$$
\begin{aligned}
\mathbb{N}_{+}^{2}\left\{x^{c-1} y^{d-1}\right\} & =\frac{u^{c-1}}{s^{c}} \Gamma(c) \cdot \frac{v^{d-1}}{p^{d}} \Gamma(d), \\
\frac{u^{c-1}}{s^{c}} \Gamma(c) \cdot \frac{v^{d-1}}{p^{d}} \Gamma(d) & =\frac{1}{u v} \int_{0}^{\infty} \int_{0}^{\infty} e^{\frac{-s x}{u}} x^{c-1} e^{\frac{-p y}{v}} y^{d-1} d x d y .
\end{aligned}
$$

Summing this result over $s$ and $p$ from one to infinity gives

$$
\begin{aligned}
u^{c} \Gamma(c) \sum_{s=1}^{\infty} \frac{1}{s^{c}} \cdot v^{d} \Gamma(d) \sum_{p=1}^{\infty} \frac{1}{p^{d}} & =\int_{0}^{\infty} x^{c-1} \sum_{s=1}^{\infty} e^{\frac{-s x}{u}} d x \int_{0}^{\infty} \sum_{p=1}^{\infty} y^{d-1} e^{\frac{-p y}{v}} d y \\
& =\int_{0}^{\infty} x^{c-1} \frac{d x}{e^{\frac{x}{u}}-1} \int_{0}^{\infty} y^{d-1} \frac{d y}{e^{\frac{y}{v}}-1}, \\
u^{c} v^{d} \Gamma(c) \zeta(s) . \Gamma(d) \zeta(p) & =\int_{0}^{\infty} x^{c-1} \frac{d x}{e^{\frac{x}{u}}-1} \int_{0}^{\infty} y^{d-1} \frac{d y}{e^{\frac{y}{v}}-1}, \\
\zeta(s) . \zeta(p) & =\frac{1}{u^{c} \Gamma(c)} \int_{0}^{\infty} x^{c-1} \frac{d x}{e^{\frac{x}{u}}-1} \cdot \frac{1}{v^{d} \Gamma(d)} \int_{0}^{\infty} y^{d-1} \frac{d y}{e^{\frac{y}{v}}-1} .
\end{aligned}
$$

This is a double integral representation for product of two zeta functions.

4. Let $f(x, t)=e^{a x+b y}$, where $a, b$ are any constants, then

$$
\mathbb{N}_{+}^{2}\{f(x, y)\}=\frac{1}{u v} \int_{0}^{\infty} \int_{0}^{\infty} e^{-\left(\frac{s x}{u}+\frac{p y}{v}\right)} e^{a x+b y} d x d y=\left(\frac{1}{s-a u}\right)\left(\frac{1}{p-b v}\right) .
$$

5. Let $f(x, t)=e^{[i(a x+b y)]}$, where $a, b$ are any constants, then

$$
\begin{aligned}
\mathbb{N}_{+}^{2}\{f(x, y)\}=\mathbb{N}_{+}^{2}\left\{e^{[i(a x+b y)]}\right\}=\frac{1}{(s-i a u)(p-i b v)} & =\frac{(s+i a u)(p+i b v)}{\left(s^{2}+a^{2} u^{2}\right)\left(p^{2}+b^{2} v^{2}\right)} \\
& =\frac{(s p-a b u v)+i(s b v+p a u)}{\left(s^{2}+a^{2} u^{2}\right)\left(p^{2}+b^{2} v^{2}\right)}
\end{aligned}
$$

Consequently,

$$
\begin{aligned}
& \mathbb{N}_{+}^{2}\{\cos (a x+b y)\}=\frac{(s p-a b u v)}{\left(s^{2}+a^{2} u^{2}\right)\left(p^{2}+b^{2} v^{2}\right)} \\
& \mathbb{N}_{+}^{2}\{\sin (a x+v y)\}=\frac{(s b v+p a u)}{\left(s^{2}+a^{2} u^{2}\right)\left(p^{2}+b^{2} v^{2}\right)}
\end{aligned}
$$


6. Let $f(x, t)=\cosh (a x+b y)$, where $a, b$ are any constants, then

$$
\begin{aligned}
\mathbb{N}_{+}^{2}\{\cosh (a x+b y)\} & =\frac{1}{2}\left[\mathbb{N}_{+}^{2}\left\{e^{a x+b y}\right\}+\mathbb{N}_{+}^{2}\left\{e^{-(a x+b y)}\right\}\right] \\
& =\frac{1}{2}\left[\frac{1}{(s-a u)(p-b v)}+\frac{1}{(s+a u)(p+b v)}\right] .
\end{aligned}
$$

Similarly, we can obtain

$$
\mathbb{N}_{+}^{2}\{\sinh (a x+b y)\}=\frac{1}{2}\left[\frac{1}{(s-a u)(p-b v)}-\frac{1}{(s+a u)(p+b v)}\right] .
$$

Now, we demonstrate some of main properties of double Natural transform as the following section.

\section{Fundamental properties of double Natural transform of partial derivatives}

If $f(x, y)$ and $g(x, y)$ are functions whose the double Natural transform exists, then we can reach the next general properties of double Natural transform under suitable conditions by applying definition of double Natural transform and integral calculus as follows.

1. Linearity property is achieved, for any constants $a, b$ such that

$$
\mathbb{N}_{+}^{2}\{a f(x, y)+b g(x, y)\}=a \mathbb{N}_{+}^{2}\{f(x, y)\}+b \mathbb{N}_{+}^{2}\{g(x, y)\} .
$$

2. Let $\mathbb{N}_{+}^{2}\{f(x, y)\}=R_{+}^{2}[(s, p) ;(u, v)]$ then

$$
\mathbb{N}_{+}^{2}\left\{e^{-a x-b y} f(x, y)\right\}=R_{+}^{2}[f(x, y) ;(s+a, p+b) ;(u, v)] .
$$

3. If $R_{+}[f(x) ; s, u]$ denoted to Natural transform of a function $f(x)$ and $R_{+}[g(x) ; p, v]$ is Natural transform of a function $g(x)$, then

$$
\mathbb{N}_{+}^{2}\{f(a x) g(b y)\}=\frac{1}{a u b v} R_{+}\left[f(x) ; \frac{s}{a}, u\right] R_{+}\left[g(x) ; \frac{p}{b}, v\right], a, b>0 .
$$

4. $\mathbb{N}_{+}^{2}\left\{\frac{\partial f(x, y)}{\partial x}\right\}=\frac{s}{u} \mathbb{N}_{+}^{2}\{f(x, y)\}-\frac{\mathbb{N}_{+}^{2}\{f(0, y)\}}{u}$.

5. $\mathbb{N}_{+}^{2}\left\{\frac{\partial^{2} f(x, y)}{\partial x^{2}}\right\}=\frac{s^{2}}{u^{2}} \mathbb{N}_{+}^{2}\{f(x, y)\}-\frac{s}{u^{2}} \mathbb{N}_{+}^{2}\{f(0, y)\}-\frac{1}{u} \mathbb{N}_{+}^{2}\left\{\frac{\partial f(0, y)}{\partial x}\right\}$.

6. $\mathbb{N}_{+}^{2}\left\{\frac{\partial f(x, y)}{\partial y}\right\}=\frac{p}{v} \mathbb{N}_{+}^{2}\{f(x, y)\}-\frac{\mathbb{N}_{+}^{2}\{f(x, 0)\}}{v}$.

7. $\mathbb{N}_{+}^{2}\left\{\frac{\partial^{2} f(x, y)}{\partial y^{2}}\right\}=\frac{p^{2}}{v^{2}} \mathbb{N}_{+}^{2}\{f(x, y)\}-\frac{p}{v^{2}} \mathbb{N}_{+}^{2}\{f(x, 0)\}-\frac{1}{v} \mathbb{N}_{+}^{2}\left\{\frac{\partial f(x, 0)}{\partial y}\right\}$.

8. $\mathbb{N}_{+}^{2}\left\{\frac{\partial^{2} f(x, y)}{\partial x \partial y}\right\}=\frac{f(0,0)}{u v}-\frac{p}{v} \mathbb{N}_{+}^{2}\{f(0, y)\}-\frac{s}{u} \mathbb{N}_{+}^{2}\{f(x, 0)\}+\mathbb{N}_{+}^{2}\{f(x, y)\}$.

9. $\frac{\partial^{n+m}}{\partial s^{n} \partial p^{m}} \mathbb{N}_{+}^{2}\{f(x, y)\}=\frac{(-1)^{m+n}}{u^{n} v^{m}} \mathbb{N}_{+}^{2}\left\{x^{n} y^{m} f(x, y)\right\}$.

Theorem 4.1. If the double Natural transform of a function $f(x, y)$ exists, then

$$
\mathbb{N}_{+}^{2}\{f(x-\eta, y-\theta) H(x-\eta, y-\theta)\}=e^{-\left(\frac{s \eta}{u}+\frac{p \theta}{v}\right)} \mathbb{N}_{+}^{2}\{f(x-\eta, y-\theta)\},
$$

where $\mathrm{H}(\mathrm{x}, \mathrm{y})$ is Heaviside unit step function defined by $\mathrm{H}(\mathrm{x}-\eta, \mathrm{y}-\theta)=1$, when $\mathrm{x}>\eta$ and $\mathrm{y}>\theta$; and $\mathrm{H}(x-\eta, y-\theta)=0$, when $x<\eta$ and $y<\theta$. 
Proof. We have by definition,

$$
\begin{aligned}
\mathbb{N}_{+}^{2}\{f(x-\eta, y-\theta) H(x-\eta, y-\theta)\} & =\frac{1}{u v} \int_{0}^{\infty} \int_{0}^{\infty} e^{-\left(\frac{s x}{u}+\frac{p y}{v}\right)} f(x-\eta, y-\theta) H(x-\eta, y-\theta) d x d y \\
& =\frac{1}{u v} \int_{0}^{\eta} \int_{0}^{\theta} e^{-\left(\frac{s x}{u}+\frac{p y}{v}\right)} f(x-\eta, y-\theta) d x d y, \quad x>\eta, y>\theta .
\end{aligned}
$$

By putting $\alpha=x-\eta, \beta=y-\theta$,

$$
\mathbb{N}_{+}^{2}\{f(\alpha, \beta)\}=\frac{e^{-\left(\frac{s \eta}{u}+\frac{p \theta}{v}\right)}}{u v} \int_{0}^{\infty} \int_{0}^{\infty} e^{-\left(\frac{s \alpha}{u}+\frac{p \beta}{v}\right)} f(\alpha, \beta) d \alpha d \beta=e^{-\left(\frac{s \eta}{u}+\frac{p \theta}{v}\right)} \mathbb{N}_{+}^{2}\{f(\alpha, \beta)\} .
$$

Theorem 4.2. If the double Natural transform of the $f(x, y)$ exists where $f(x, y)$ is a periodic function of periods a and $b$ (that is $f(x+a, y+b)=f(x, y), \forall x, y)$, then

$$
\mathbb{N}_{+}^{2}\{f(x, y)\}=\frac{\left[1-e^{-\left(\frac{a s}{u}+\frac{b p}{v}\right)}\right]^{-1}}{u v} \int_{0}^{a} \int_{0}^{b} e^{-\left(\frac{s x}{u}+\frac{p y}{v}\right)} d x d y .
$$

Proof.

$$
\begin{aligned}
\mathbb{N}_{+}^{2}\{f(x, y)\} & =\frac{1}{u v} \int_{0}^{\infty} \int_{0}^{\infty} e^{-\left(\frac{s x}{u}+\frac{p y}{v}\right)} f(x, y) d x d y \\
& =\frac{1}{u v} \int_{0}^{a} \int_{0}^{b} e^{-\left(\frac{s x}{u}+\frac{p y}{v}\right)} f(x, y) d x d y+\frac{1}{u v} \int_{a}^{\infty} \int_{b}^{\infty} e^{-\left(\frac{s x}{u}+\frac{p y}{v}\right)} f(x, y) d x d y .
\end{aligned}
$$

Setting $x=\alpha+a, y=\beta+b$ in the second double integral, we have

$$
\begin{aligned}
\mathbb{N}_{+}^{2}\{f(x, y)\} & =\frac{1}{u v} \int_{0}^{a} \int_{0}^{b} e^{-\left(\frac{s x}{u}+\frac{p y}{v}\right)} f(x, y) d x d y+\frac{e^{-\left(\frac{a s}{u}+\frac{b p}{v}\right)}}{u v} \int_{0}^{\infty} \int_{0}^{\infty} e^{-\left(\frac{s \alpha}{u}+\frac{p \beta}{v}\right)} f(\alpha+a, \beta+b) d \alpha d \beta \\
& =\frac{1}{u v} \int_{0}^{a} \int_{0}^{b} e^{-\left(\frac{s x}{u}+\frac{p y}{v}\right)} f(x, y) d x d y+\frac{e^{-\left(\frac{a s}{u}+\frac{b p}{v}\right)}}{u v} \int_{0}^{\infty} \int_{0}^{\infty} e^{-\left(\frac{s \alpha}{u}+\frac{p \beta}{v}\right)} f(\alpha, \beta) d \alpha d \beta \\
& =\frac{1}{u v} \int_{0}^{a} \int_{0}^{b} e^{-\left(\frac{s x}{u}+\frac{p y}{v}\right)} f(x, y) d x d y+e^{-\left(\frac{a s}{u}+\frac{b p}{v}\right)} \mathbb{N}_{+}^{2}\{f(x, y)\} .
\end{aligned}
$$

Consequently,

$$
\mathbb{N}_{+}^{2}\{f(x, y)\}=\frac{\left[1-e^{-\left(\frac{a s}{u}+\frac{b p}{v}\right)}\right]^{-1}}{u v} \int_{0}^{a} \int_{0}^{b} e^{-\left(\frac{s x}{u}+\frac{p y}{v}\right)} d x d y .
$$

This proves the theorem of double Natural transform of a periodic function.

\section{Convolution theorem and relationship with other transforms}

In this section, convolution theorem and double Natural transform of derivative of the convolution are presented. Further we produce the connection between double Natural transform and double Laplace, double Sumudu transforms.

Theorem 5.1. Suppose that $\mathrm{f}(\mathrm{x}, \mathrm{y})$ and $\mathrm{g}(\mathrm{x}, \mathrm{y})$ have double Natural transforms, thus

$$
\mathbb{N}_{+}^{2}\{(f * * g)(u, v) ;(s, p)\}=u v \mathbb{N}_{+}^{2}\{f(u, v) ;(s, p)\} \mathbb{N}_{+}^{2}\{g(u, v) ;(s, p)\} .
$$


Proof. By applying definition of double Natural transform and double convolution, it yields that

$$
\begin{aligned}
\mathbb{N}_{+}^{2}\{(f * * g)(x, y)\} & =\frac{1}{u v} \int_{0}^{\infty} \int_{0}^{\infty} e^{-\left(\frac{s x}{u}+\frac{p y}{v}\right)}(f * * g)(x, y) d x d y \\
& =\frac{1}{u v} \int_{0}^{\infty} \int_{0}^{\infty} e^{-\left(\frac{s x}{u}+\frac{p y}{v}\right)}\left(\int_{0}^{y} \int_{0}^{x} f(\alpha, \beta) g(x-\alpha, y-\beta) d \alpha d \beta\right) d x d y,
\end{aligned}
$$

let $\eta=x-\alpha, \zeta=y-\beta$, and extend upper bound of integrals to $x \longrightarrow \infty, y \longrightarrow \infty$, it implies that

$$
\mathbb{N}_{+}^{2}\{(f * * g)(x, y)\}=\frac{1}{u v} \int_{0}^{\infty} \int_{0}^{\infty} e^{-\left(\frac{s \alpha}{u}+\frac{p \beta}{v}\right)} f(\alpha, \beta) d \alpha d \beta \int_{-\eta}^{\infty} \int_{-\zeta}^{\infty} e^{-\left(\frac{s \eta}{u}+\frac{p \zeta}{v}\right)} g(\eta, \zeta) d \eta d \zeta,
$$

the functions $f(x, y), g(x, y)$ have zero value for $x<0$, and $y<0$, therefore

$$
\mathbb{N}_{+}^{2}\{(f * * g)(x, y)\}=\frac{1}{u v} \int_{0}^{\infty} \int_{0}^{\infty} e^{-\left(\frac{s \alpha}{u}+\frac{p \beta}{v}\right)} f(\alpha, \beta) d \alpha d \beta \int_{0}^{\infty} \int_{0}^{\infty} e^{-\left(\frac{s \eta}{u}+\frac{p \zeta}{v}\right)} g(\eta, \zeta) d \eta d \zeta,
$$

then

$$
\mathbb{N}_{+}^{2}\{(f * * g)(u, v) ;(s, p)\}=u v \mathbb{N}_{+}^{2}\{f(u, v) ;(s, p)\} \mathbb{N}_{+}^{2}\{g(u, v) ;(s, p)\}
$$

Moreover, double Natural transform of derivative of double convolution can be obtained by

or

$$
\mathbb{N}_{+}^{2}\left\{\frac{\partial}{\partial x}(f * * g)(x, y)(u, v) ;(s, p)\right\}=u v \mathbb{N}_{+}^{2}\left\{\frac{\partial}{\partial x} f(x, y)(u, v) ;(s, p)\right\} \mathbb{N}_{+}^{2}\{g(x, y)(u, v) ;(s, p)\}
$$

$$
=u v \mathbb{N}_{+}^{2}\{f(x, y)(u, v) ;(s, p)\} \mathbb{N}_{+}^{2}\left\{\frac{\partial}{\partial x} g(x, y)(u, v) ;(s, p)\right\} .
$$

Next we derive the connection between double Natural and double Laplace, double Sumudu transforms in successive theorems.

Theorem 5.2 (Double Natural-Laplace duality). If the double Natural transform of a function $f(x, y) \in \mathbb{R}_{+}^{2}$ exists, then

$$
\mathbb{N}_{+}^{2}\{f(x, y)(u, v) ;(s, p)\}=\frac{1}{u v} F^{2}\left(\frac{u}{s}, \frac{v}{p}\right),
$$

where $\mathrm{F}^{2}$ denotes the double Laplace transform of the function $\mathrm{f}$.

Proof. The result can get directly from the formula (3.2) such that

$$
\mathbb{N}_{+}^{2}\{f(x, y)\}=\frac{1}{u v} \int_{0}^{\infty} \int_{0}^{\infty} e^{-\left(\frac{s x}{u}+\frac{p y}{v}\right)} f(x, y) d x d y=\frac{1}{u v} F^{2}\left(\frac{u}{s}, \frac{v}{p}\right)
$$

Theorem 5.3 (Double Natural-Sumudu duality). If the double Natural transform of a function $f(x, y) \in \mathbb{R}_{+}^{2}$ exists and $\mathrm{G}^{2}$ denotes the double Sumudu transform of $\mathrm{f}(\mathrm{x}, \mathrm{y})$, then

$$
\mathbb{N}_{+}^{2}\{f(x, y)(u, v) ;(s, p)\}=\frac{1}{s p} G^{2}\left(\frac{s}{u}, \frac{p}{v}\right) .
$$

Proof. Substituting $w=s x, \tau=$ py in the formula (3.1), then we obtain

$$
\mathbb{N}_{+}^{2}\{f(x, y)\}=\frac{1}{s p} \int_{0}^{\infty} \int_{0}^{\infty} e^{-(\omega+\tau)} f\left(\frac{u}{s} \omega, \frac{v}{p} \tau\right) d \omega d \tau=\frac{1}{s p} G^{2}\left(\frac{u}{s}, \frac{v}{p}\right) .
$$




\section{Applications}

In the following examples we denote double Natural transform of the function $w(x, y)$ by

$$
W[(s, p) ;(u, v)] .
$$

Example 6.1. Consider the homogeneous telegraphic equation given by

$$
w_{x x}(x, y)-w_{y y}(x, y)-w_{y}(x, y)-w(x, y)=0
$$

with boundary conditions

$$
w(0, y)=e^{-y}, w_{x}(0, y)=e^{-y}
$$

and initial conditions

$$
w(x, 0)=e^{x}, w_{y}(x, 0)=-e^{x} .
$$

Proof. By applying double Natural transform on the equation (6.1) and single Natural transform on the equations (6.2) and (6.3), we obtain

$$
W[(s, p) ;(u, v)]=\frac{u^{2} v^{2}\left(s^{2} v^{2}-u^{2} p^{2}-p u v^{2}-u^{2} v^{2}\right)}{u^{2} v^{2}(p+v)(s-u)\left(s^{2} v^{2}-u^{2} p^{2}-p u v^{2}-u^{2} v^{2}\right)}=\frac{1}{(p+v)(s-u)}
$$

Taking double inverse Natural transform for the equation (6.4), then we get the same solution that is given by using double Laplace transform (see [16]),

$$
w(x, y)=e^{x-y}
$$

In the following example, we apply double Natural transform on non-homogeneous telegraph equation as follows.

Example 6.2. Suppose the non-homogeneous telegraph equation that is given as

$$
w_{x x}(x, y)-w_{y y}(x, y)-w_{y}(x, y)-w(x, y)=-2 e^{x+y}
$$

with boundary conditions

$$
w(0, y)=e^{y}, w_{x}(0, y)=e^{y},
$$

and initial conditions

$$
w(x, 0)=e^{x}, w_{y}(x, 0)=e^{x} .
$$

Proof. By applying double Natural transform on the equation (6.5) and single Natural transform on the equations (6.6) and (6.7), we obtain

$$
W[(s, p) ;(u, v)]=\frac{u^{2} v^{2}\left(s^{2} v^{2}-u^{2} p^{2}-p u v^{2}-u^{2} v^{2}\right)}{u^{2} v^{2}(p+v)(s-u)\left(s^{2} v^{2}-u^{2} p^{2}-p u v^{2}-u^{2} v^{2}\right)}=\frac{1}{(p-v)(s-u)} .
$$

Taking double inverse Natural transform for the equation (6.8), then we have the same solution that is obtained by using double Laplace transform (see [16]),

$$
w(x, y)=e^{x+y} \text {. }
$$

Example 6.3. Consider the following wave equation as

$$
w_{x x}(x, y)-w_{y y}(x, y)=3\left(e^{2 x+y}-e^{x+2 y}\right), x, y \in \mathbb{R}_{+},
$$


with boundary conditions

$$
w(0, y)=e^{y}+e^{2 y}, \quad w_{x}(0, y)=2 e^{y}+e^{2 y}
$$

and initial conditions

$$
w(x, 0)=e^{2 x}+e^{x}, \quad w_{y}(x, 0)=e^{2 x}+2 e^{x} .
$$

Proof. Taking double Natural transform of the equation (6.9) and single Natural transform of conditions (6.10) and (6.11), then we obtain

$$
\begin{aligned}
\frac{s^{2} v^{2}-p^{2} u^{2}}{u^{2} v^{2}} W[(s, p) ;(u, v)]= & \frac{1}{u^{2} v^{2}}\left[\frac{v^{2}\left(s^{2}-4 u^{2}\right)-u^{2}\left(p^{2}-v^{2}\right)}{(s-2 u)(p-v)}+\frac{v^{2}\left(s^{2}-u^{2}\right)-u^{2}\left(p^{2}-4 v^{2}\right)}{(s-u)(p-2 v)}\right] \\
& +\frac{3}{(s-2 u)(p-v)}-\frac{3}{(s-u)(p-2 v)},
\end{aligned}
$$

after algebraic operations, it implies that

$$
W[(s, p) ;(u, v)]=\frac{1}{(s-2 u)(p-v)}+\frac{1}{(s-u)(p-2 v)} .
$$

Taking double inverse Natural transform for the equation (6.12), we get the following solution

$$
w(x, 0)=e^{2 x+y}+e^{x+2 y} .
$$

In the next example, double Natural transform is applied on partial integro-differential equation as follows.

Example 6.4. Assume that partial integro-differential equation defined as follows

$$
w_{x x}(x, y)-w_{y y}(x, y)+w(x, y)+\int_{0}^{y} \int_{0}^{x} e^{x-\alpha+y-\beta} w(\alpha, \beta) d \alpha d \beta=f(x, y)
$$

with boundary conditions

$$
w(x, 0)=e^{x}, w_{y}(x, 0)=e^{x},
$$

and initial conditions

$$
w(0, y)=e^{y}, w_{x}(0, y)=e^{y} .
$$

Proof. By applying double Natural transform on the equation (6.13) and single Natural transform on the equations (6.14) and (6.15), we obtain

$$
\begin{aligned}
\left(\frac{s^{2}}{u^{2}}-\frac{p^{2}}{v^{2}}+\frac{u v}{(s-u)(p-v)}+1\right) W[(s, p) ;(u, v)] & =\frac{1}{(s-u)(p-v)}+\frac{u v}{(s-u)^{2}(p-v)^{2}} \\
& +\frac{s}{u^{2}(p-v)}+\frac{1}{u(p-v)}-\frac{p}{v^{2}(s-u)}-\frac{1}{v(s-u)}, \\
\left(\frac{s^{2}}{u^{2}}-\frac{p^{2}}{v^{2}}+\frac{u v}{(s-u)(p-v)}+1\right) W[(s, p) ;(u, v)] & =\frac{1}{(s-u)(p-v)}\left(\frac{s^{2}}{u^{2}}-\frac{p^{2}}{v^{2}}+\frac{u v}{(s-u)(p-v)}+1\right), \\
W[(s, p) ;(u, v)] & =\frac{1}{(s-u)(p-v)} .
\end{aligned}
$$

Taking double inverse Natural transform for the equation (6.16), we get the following solution

$$
w(x, y)=e^{x+y} .
$$

The solution $w(x, y)$ is in agreement with the result that got in [16]. 


\section{Conclusion}

This work deals with definition of double Natural transform and its inverse. Fundamental properties of double Natural transform are obtained. Further, some examples and applications on double Natural transform are presented, and the results are in agreement with [16]. Using double Natural transform to solve some types of equations with variable coefficients will be a future work.

\section{Acknowledgment}

The authors would like to thank the referees for valuable suggestions and comments, which helped the authors to improve this article substantially.

\section{References}

[1] S. K. Q. Al-Omari, On the application of natural transforms, Inter. J. Pure Appl. Math., 85 (2013), 729-744. 1

[2] M. A. Asiru, Sumudu transform and the solution of integral equations of convolution type, Internat. J. Math. Ed. Sci. Tech., 32 (2001), 906-910. 1

[3] F. B. M. Belgacem, Introducing and analysing deeper Sumudu properties, Nonlinear Stud., 13 (2006), 23-41. 1

[4] F. B. M. Belgacem, Sumudu transform applications to Bessel functions and equations, Appl. Math. Sci. (Ruse), 4 (2010), 3665-3686.

[5] F. B. M. Belgacem, A. A. Karaballi, Sumudu transform fundamental properties investigations and applications, J. Appl. Math. Stoch. Anal., 2006 (2006), 23 pages.

[6] F. B. M. Belgacem, A. A. Karaballi, S. L. Kalla, Analytical investigations of the Sumudu transform and applications to integral production equations, Math. Probl. Eng., 2003 (2003), 103-118. 1

[7] F. B. M. Belgacem, R. Silambarasan, Advances in the natural transform, AIP Conf. Proc., 1493 (2012), 106-110. 1

[8] F. B. M. Belgacem, R. Silambarasan, Maxwell's equations solutions by means of the natural transform, Int. J. Math. Eng. Sci. Aerosp., 3 (2012), 313-323. 1

[9] F. B. M. Belgacem, R. Silambarasan, Theory of natural transform, Int. J. Math. Eng. Sci. Aerosp., 3 (2012), 99-124. 1, 2.6

[10] Y. A. Brychkov, H.-J. Glaeske, A. P. Prudnikov, Vũ Kim Tuân, Multidimensional integral transformations, Gordon and Breach Science Publishers, Philadelphia, PA, (1992). 1

[11] V. A. Ditkin, A. P. Prudnikov, Operational calculus in two variables and its applications, Translated by D. M. G. Wishart, International Series of Monographs on Pure and Applied Mathematics, Pergamon Press, New YorkOxford-London-Paris, (1962). 2.5

[12] D. G. Duffy, Transform methods for solving partial differential equations, Second edition, Chapman \& Hall/CRC, Boca Raton, FL, (2004). 1

[13] H. Eltayeb, A. K1lıcman, A note on solutions of wave, Laplace's and heat equations with convolution terms by using a double Laplace transform, Appl. Math. Lett., 21 (2008), 1324-1329. 1

[14] H. Eltayeb, A. Kılıçman, A note on the Sumudu transforms and differential equations, Appl. Math. Sci. (Ruse), 4 (2010), 1089-1098. 1

[15] H. Eltayeb, A. Kılıçman, On double Sumudu transform and double Laplace transform, Malays. J. Math. Sci., 4 (2010), 17-30. 1

[16] H. Eltayeb, A. Kılıçman, A note on double Laplace transform and telegraphic equations, Abstr. Appl. Anal., 2013 (2013), 6 pages. $1,6,6,6,7$

[17] T. A. Estrin, T. J. Higgins, The solution of boundary value problems by multiple Laplace transformations, J. Franklin Inst., 252 (1951), 153-167. 1, 2.2, 2.5

[18] Z. H. Khan, W. A. Khan, N-transform properties and applications, NUST J. Eng. Sci. (NJES), 1 (2008), 127-133. 1, 2.6

[19] A. Kılıçman, H. Eltayeb, A note on integral transforms and partial differential equations, Appl. Math. Sci. (Ruse), 4 (2010), 109-118. 1

[20] A. Kılıçman, H. Eltayeb, On a new integral transform and differential equations, Math. Probl. Eng., 2010 (2010), 13 pages. 1

[21] A. Kılıçman, H. Eltayeb, Some remarks on the Sumudu and Laplace transforms and applications to differential equations, ISRN Appl. Math., 2012 (2012), 13 pages. 1

[22] A. Kılıçman, H. Eltayeb, R. P. Agarwal, On Sumudu transform and system of differential equations, Abstr. Appl. Anal., 2010 (2010), 11 pages. 1

[23] A. Kılıçman, H. E. Gadain, An application of double Laplace transform and double Sumudu transform, Lobachevskii J. Math., 30 (2009), 214-223. 1

[24] A. Kılıçman, H. E. Gadain, On the applications of Laplace and Sumudu transforms, J. Franklin Inst., 347 (2010), 848 862. 1 
[25] M. Omran, A. Kılıçman, Natural transform of fractional order and some properties, Cogent Math., 3 (2016), 1-8. 1

[26] R. Silambarasan, F. B. M. Belgacem, Applications of the natural transform to Maxwells equations, Prog. Electromagnetic Research Symposium Proc. Suzhou, China, (2011), 899-902. 1

[27] I. N. Sneddon, The use of integral transforms, McGraw-Hill, New York, (1972). 2.2

[28] M. R. Spiegel, Schaum's outline of theory and problems of Laplace transforms, McGraw-Hill, New York, (1965). 1, 2.1

[29] J. M. Tchuenche, N. S. Mbare, An application of the double Sumudu transform, Appl. Math. Sci. (Ruse), 1 (2007), 31-39. 1, 2.4

[30] G. K. Watugala, Sumudu transform: a new integral transform to solve differential equations and control engineering problems, Internat. J. Math. Ed. Sci. Tech., 24 (1993), 35-43. 1

[31] G. K. Watugala, The Sumudu transform for functions of two variables, Math. Engrg. Indust., 8 (2002), 293-302. 2.3 